\title{
Abundance and evolution of galaxy clusters in cosmological models with massive neutrino
}

\author{
N. A. Arhipova ${ }^{1}$, T. Kahniashvili ${ }^{2,3}$, and V. N. Lukash ${ }^{1}$ \\ 1 Astro Space Center of P.N. Lebedev Physical Institute, Moscow, Russia \\ 2 Center for Plasma Astrophysics, Abastumani Astrophysical Observatory, Tbilisi, Georgia \\ 3 Department of Physics and Astronomy, Rutgers University, Piscataway, NJ, USA
}

Received 3 August 2001 / Accepted 11 January 2002

\begin{abstract}
The time evolution of the number density of galaxy clusters and their mass and temperature functions are used to constrain cosmological parameters in the spatially flat dark matter models containing hot particles (massive neutrino) as well as cold and baryonic matter. We test the modified MDM $(\Lambda=0)$ models with cosmic gravitational waves and show that they neither pass the cluster evolution test nor reproduce the observed height of the first acoustic peak in $\Delta T / T$ spectrum, and therefore should be ruled out. The models with a non-zero cosmological constant are in better agreement with observations. We estimate the free cosmological parameters in $\Lambda \mathrm{MDM}$ with a negligible abundance of gravitational waves, and find that within the parameter ranges $h \in(0.6,0.7), n \in(0.9,1.1), f_{\nu} \equiv \Omega_{\nu} / \Omega_{\mathrm{m}} \in(0,0.2)$, (i) the value of $\Omega_{\Lambda}$ is strongly affected by a small fraction of hot dark matter: $0.45<\Omega_{\Lambda}<0.7(1 \sigma \mathrm{CL})$, and (ii) the redshift evolution of galaxy clusters alone reveals the following explicit relation between $\Omega_{\Lambda}$ and $f_{\nu}$ :

$\Omega_{\Lambda}+0.5 f_{\nu}=0.65 \pm 0.1$.

This degeneracy is also expected in LSS tests (with a smaller error). The present accuracy of observational data allows to bound the fraction of hot matter, $f_{\nu} \in(0,0.2)$; the number of massive neutrino species remains undelimited, $N_{\nu}=1,2,3$.
\end{abstract}

Key words. cosmology: observations - cosmology: theory - large-scale structure of the Universe clusters of galaxies

\section{Introduction}

One of the most important problems of modern cosmology is the formation of large-scale structure in the Universe (LSS). In the last decade progress in observations of LSS and cosmic microwave background anisotropy (CMBA) has allowed a productive comparison of theory with observations. Any realistic cosmological model should be consistent with LSS observational data in the range of scales from sub-Mpc (Gnedin 1998; Peackock 1997) up to the cosmological horizon (Smoot et al. 1992; Jaffe et al. 2001).

According to the standard theory of gravitational instability the observable LSS in the Universe has been formed by growth of small density inhomogeneities generated during the very early stages of cosmological expansion. Two main features of the primordial perturbation field - the Gaussian character of linear density perturbations and the bottom-up LSS formation - are confirmed

Send offprint requests to: N. A. Arhipova,

e-mail: arna@lukash.asc.rssi.ru by available astrophysical observations. Therefore, assuming that the initial conditions are given one can derive the theoretical predictions for LSS formation in a dark matter (DM) model and then use statistical analysis and observational data to test the viability of the considered models, i.e. to constrain the allowed range of principal cosmological parameters.

Nowadays, the most popular cosmological models are cold DM with non-zero cosmological constant ( $\Lambda \mathrm{CDM})$ (Peebles 1984; Kofman \& Starobinsky 1985) and mixed DM without (MDM) (Fang et al. 1984; Shafi \& Stecker 1984; Valdarnini \& Bonometto 1984; Lukash 1991; Lucchin et al. 1996) and with ( $\mathrm{MDM}$ ) (Kahniashvili et al. 1996; Novosyadlyj et al. 1999b and Refs. therein) cosmological constant, where MDM is in the form of nonbaryonic cold (neutralino or hypothetical axions) and hot (massive neutrino) particles.

The cosmological impact of non-baryonic collisionless matter depends on the free-streaming path of DM particles: at small scales $\left(k>k_{\mathrm{FS}}\right)$ the perturbations smooth 
out, whereas at large scales $\left(k<k_{\mathrm{FS}}\right)$ they grow to form the gravitationally bounded objects of mass greater than $M_{\mathrm{FS}}$ (Novikov \& Zel'dovich 1975). Due to the various freestreaming pathes of cold and hot particles $\left(k_{\mathrm{FS}}^{(\mathrm{c})} \gg k_{\mathrm{FS}}^{(\nu)}\right)$ the growth rate of density perturbations is different in CDM and HDM components. The linear perturbation power spectrum formed by redshift $z, P(k, z)$, depends strongly on the abundance of each component $\left(\Omega_{\mathrm{c}}, \Omega_{\nu}\right)$ and is given by the production of the initial power spectrum, $P_{0}(k)$, the transfer function, $T(k, z)$, and the perturbation growth rate, $D(z)$ (e.g. Padmanabhan 1993). Both the transfer function and the growth factor are DM dependent and describe the evolution of initial density perturbations during expansion of the Universe (Zakharov 1979; Bardeen et al. 1986; Holtzman 1989; Eisenstein \& Hu 1998; Novosyadly et al. 1999a).

All cosmological models have been re-addressed after CMBA experimental detection (Bennett et al. 1996) to reveal their positive and negative features.

Both $\Lambda$ CDM and MDM models have met several difficulties.

As far as $\Lambda$ CDM models are concerned (Kofman et al. 1993; Eke et al. 1996; Liddle et al. 1996a,b; Primack \& Klypin 1996) they demand a high value of the cosmological constant $\left(\Omega_{\Lambda} \geq 0.7\right)$. In this case $\Lambda$ CDM is able to fit a set of LSS observational constraints whereas at small scales it overproduces the number of collapsed objects by a factor of 2 in comparison with the corresponding number of gravitationally bounded objects in galactic cataloges (Klypin et al. 1996; Gawiser 2000; Bond et al. 2000).

Regarding MDM models the difficulties are related to late galaxy (quasar) formation (Pogosyan \& Starobinsky 1995; Komberg et al. 1996) and too high numbers of obtained galaxy clusters (Ma 1996; Valdarnini et al. 1998; Gardini et al. 1999; Rahman \& Shandarin 2001). Standard MDM with one, two or three species of massive neutrino are ruled out at $2 \sigma$ CL (Novosyadlyj et al. 1999b).

A way to overcome the sMDM difficulties could be the consideration of decaying neutrinos (Bonometto \& Pierpaoli 1998), or models with cosmic gravitational waves (CGW) (Arkhipova et al. 1999; Melchiorri et al. 1999), or a non-zero cosmological constant $(\Lambda \mathrm{MDM})$ (Kahniashvili et al. 1996; Tegmark 1999). In this paper we consider the models with stable neutrinos.

An importance of fundamental CGW has been emphasized by Starobinsky (1979), Rubakov et al. (1982), Lukash \& Mikheeva (2000). Theoretical predictions for cluster abundance in MDM models with CGW has been presented by Ma (1996) for the red spectra of density perturbations $(n<1)$, and by Mikheeva et al. (2001) for both blue $(n>1)$ and red $(n<1)$ scalar perturbation spectra. Although $\Lambda$ CDM models with $\Omega_{\mathrm{m}} \leq 0.3$ normalized by COBE 4-year data (Bunn \& White 1997) are consistent with the cluster number density test, in order to archive an agreement with CMBA and cluster abundance data in MDM models it is necessary to take into account the CGW contribution in the derived value of $\Delta T / T$ at $10^{\circ}$ angular scale. The latter is estimated by parameter $T / S$, the ratio of the tensor to the scalar mode contributions.

As an alternative to $\Lambda$ CDM and MDM, the $\Lambda$ MDM models have been considered by Valdarnini et al. (1998), Novosyadlyj et al. (1999b), Primack \& Gross (2000), Andres et al. (2000). The advantage of these models is in retaining the inflationary paradigm and the flat Harrison-Zel'dovich spectrum $(n=1)$ with a smaller value (comparing to $\Lambda \mathrm{CDM}$ ) of cosmological $\Lambda$-term. Other notable features of $\Lambda \mathrm{MDM}$ are the possibility of negligible CGW contribution $(T / S=0)$ and a small fraction of hot particles: even $10 \%$ of massive neutrino in matter content $\left(\Omega_{\nu} / \Omega_{\mathrm{m}} \sim 0.1\right)$ could change the value of the cosmological constant, being however in good agreement with other independent tests: $\mathrm{CMB}$ data (Hu et al. 2000), distant SNIa (Perlmutter et al. 1999), Hipparcos data (Feast \& Catchpole 1997), QSO lensing (Kochanek 1996).

In this paper we consider MDM (with CGW) and $\Lambda \mathrm{MDM}$ (with $T / S=0$ ) applying to these models the cluster abundance and evolution tests. The evolution test constrains most efficiently the parameter $\Omega_{\mathrm{m}}$, and hence $\Omega_{\Lambda}$ in spatially flat cosmological models (e.g. Bahcall et al. 1997; Donahue \& Voit 1999; Henry 2000). Our aim is to demonstrate how the presence of hot matter influences the estimation of $\Omega_{\Lambda}, h$, and other cosmological parameters. Here, instead of doing an exact $\chi^{2}$ analysis, we rather look for the tendencies and correlations between cosmological parameters when introducing a fraction of massive neutrino in the Universe.

We describe our models in Sect. 2, and the galaxy cluster tests in Sect. 3. The results are discussed in Sect. 4, with the conclusions in Sect. 5 .

\section{Cosmological models with massive neutrino}

We assume that DM is given by mixture of CDM and HDM components in the flat background space. The free model parameters are:

$-\Omega_{\mathrm{m}}$, the total matter density in the Universe $\left(\Omega_{\mathrm{m}}=1-\Omega_{\Lambda}=\Omega_{\mathrm{b}}+\Omega_{\mathrm{c}}+\Omega_{\nu}\right.$, the latter being density parameters of baryons, cold, and hot particles, respectively);

$-f_{\nu} \equiv \Omega_{\nu} / \Omega_{\mathrm{m}}$, the fraction of hot DM;

- $N_{\nu}$, the number of massive neutrino species;

$-h$, the Hubble constant in units $100 \mathrm{~km} \mathrm{~s}^{-1} \mathrm{Mpc}^{-1}$;

- $n$, the slope-index of post-inflationary density perturbation power spectrum.

The massive and massless neutrinos ${ }^{1}$ are described by the corresponding distribution functions which are evaluated from the Boltzmann-Vlasov collisionless equations.

\footnotetext{
1 All neutrinos are active, the total number of neutrino species is three. Accordingly, $N_{\nu}=1,2,3$.
} 
Cold particles are treated as a pressureless fluid $\left(p_{\mathrm{c}}=0\right)$. Baryons and photons are described as an ideal hydrodynamic fluid satisfying the Euler equations of motion. Here we choose the fixed value for baryon density parameter, $\Omega_{\mathrm{b}}=0.015 / h^{2}$. All DM components interact with each other only gravitationally.

The calculation of the perturbation dynamics (the transfer functions) demands a joint solution of the described self-consistent set of equations. It can be done numerically (Seljak \& Zaldarriaga 1996) or analytically in long and short wave regions (Zakharov 1979; Bardeen et al. 1986; Holtzman 1989), the possibility of semianalitical approximations of some transfer functions has been shown by Eisenstein \& Hu (1998), Novosyadlyj et al. (1999a).

Assuming the power low post-inflationary density perturbation spectrum, $P_{0}(k) \infty k^{n}$, the final power spectrum of total density perturbations can be expressed as

$P(k, z)=A k^{n} T^{2}(k, z)\left[\frac{D(z)}{D(0)}\right]^{2}$,

where $A$ is the normalization constant, $T(k, z)$ is the total transfer function, $D(z)$ is the growth factor ${ }^{2}$,

$D(z)=\frac{g\left(\Omega_{\mathrm{m}}(z)\right)}{1+z}$,

and $g\left(\Omega_{\mathrm{m}}(z)\right)$ is the suppression coefficient (Kofman \& Starobinsky 1985).

According to Carroll et al. (1992) the function $g(x)$ can be approximated as

$g(x)=5 x\left(2 x^{\frac{4}{7}}+\frac{2+x(209-x)}{70}\right)^{-1}$,

and the current matter abundance $\Omega_{\mathrm{m}}(z)$ is written as follows:

$\Omega_{\mathrm{m}}(z)=\Omega_{\mathrm{m}} \frac{(1+z)^{3}}{1-\Omega_{\mathrm{m}}+(1+z)^{3} \Omega_{\mathrm{m}}}, \quad \Omega_{\mathrm{m}} \equiv \Omega_{\mathrm{m}}(0)$.

We use the transfer function approximations for sCDM (Bardeen et al. 1986) and MMDM (Eisenstein \& Hu 1998) models.

\section{Cluster mass and temperature functions and the evolution test}

The mass function for the gravitationally bounded halos of mass greater than $M$ formed in the flat Universe by redshift $z$ is given by (Press \& Schechter 1974)

$N(>M, z)=\int_{M}^{\infty} n\left(M^{\prime}, z\right) \mathrm{d} M^{\prime}$

${ }^{2}$ The function $D(z)$ is given as the growth rate of linear density perturbations in the model without hot matter (with the same parameters $\Omega_{\mathrm{m}}, h$, and $n$ ). where $n(M, z) \mathrm{d} M$ is the comoving number density of collapsed objects with masses lying in the interval $(M, M+\mathrm{d} M)$ :

$n(M, z)=\sqrt{\frac{2}{\pi}} \frac{\rho \delta_{\mathrm{c}}}{M} \frac{1}{\sigma^{2}(R, z)}\left|\frac{\mathrm{d} \sigma(R, z)}{\mathrm{d} M}\right| \mathrm{e}^{-\frac{\delta_{c}^{2}}{2 \sigma^{2}(R, z)}}$.

$M=\frac{4}{3} \pi \rho R^{3}, \rho$ is the mean matter density, and $\delta_{\mathrm{c}}$ is the critical density contrast for a linear overdensity able to collaps. The rms amplitude of density fluctuation in the spheres of radius $R$ at redshift $z$ is related to the power spectrum as

$\sigma^{2}(R, z)=\frac{1}{2 \pi^{2}} \int_{0}^{\infty} P(k, z)|W(k R)|^{2} k^{2} \mathrm{~d} k$,

where $W(k R)$ is a Fourier component of the top-hat window function: $W(x)=\frac{3}{x^{3}}(\sin x-x \cos x)$. We denote $\sigma_{R} \equiv \sigma(R, 0)$ for $z=0$.

In the matter-dominated Universe $\delta_{\mathrm{c}}=1.686$ (Eke et al. 1996; Viana \& Liddle 1996), in the flat models with $\Lambda$-term $\delta_{\mathrm{c}}$ depends weakly on the current matter abundance (Liddle et al. 1996a; Locas \& Hoffman 2000).

The theoretical mass functions obtained with the help of the Press-Schechter formalism (Eqs. (5)-(7)) are in good agreement with other methods including numerical simulations (Efstathiou et al. 1988; Lacey \& Cole 1994; Gross et al. 1998; Eke et al. 1998; Bode et al. 2000; Wu $2000)$. Due to the exponential dependence of $n(M, 0)$ on $\sigma_{R}$ (see Eq. (6)) the cluster number density is very sensitive to the value of $\sigma_{8}$.

Rich galaxy clusters are strong X-rays sources characterised by the gas temperature. The physical mechanisms of clusters formation promp the relation between cluster mass and temperature, $T_{\mathrm{g}} \infty M^{2 / 3}$, confirmed by numerical simulations (e.g. Navarro et al. 1995). The proportionality coefficient depends slightly on cosmological model (mainly, on the parameter $\Omega_{\mathrm{m}}$ ). Here, we use the $T-M$ relation for isothermal gas given by Eke et al. (1996):

$$
\begin{aligned}
T_{\mathrm{g}}= & \frac{7.75}{\beta}\left(\frac{6.8}{5 X+3}\right) M_{15}^{\frac{2}{3}}\left(\frac{\Omega_{\mathrm{m}}}{\Omega_{\mathrm{m}}(z)}\right)^{\frac{1}{3}} \\
& \times\left(\frac{\Delta_{\mathrm{cr}}}{178}\right)^{\frac{1}{3}}(1+z) \mathrm{Kev}
\end{aligned}
$$

where $\beta(\simeq 1)$ is the ratio of the galaxy kinetic energy to the gas thermal energy, $X(\simeq 0.76)$ is the hydrogen mass fraction, and the mass $M_{15}$ is given in units $10^{15} h^{-1} M_{\odot}$. The value $\Delta_{\text {cr }}$ is the ratio of a mean halo density (within the virial radius of collapsed object) to the critical density of the Universe at corresponding redshift. For $\Omega_{\mathrm{m}} \leq 1, \Delta_{\text {cr }}$ can be derived analytically and approximated as $\Delta_{\mathrm{cr}}=$ $178 \Omega_{\mathrm{m}}^{0.45}$ for $z=0$.

As well as the cluster mass function $N(>M) \equiv$ $N(>M, 0)$, the X-cluster temperature function $N(>T) \equiv$ $N(>T, 0)$ is also sensitive to $\sigma_{R}$. The comparison between the observed temperature (Henry \& Arnaud 1991) and 
mass (Bahcall \& Cen 1993) functions shows a good agreement with Eq. (8) at $z=0$. Comparing with observational data both tests provide a powerful constraint on $\sigma$-value in different DM models (Valdarnini et al. 1998; Bahcall \& Fan 1998; Durrer \& Novosyadlyj 2001). On the other hand CMBA strongly depends on the density perturbation spectrum as well. The consistency with all these tests determines the model parameters (e.g. Bridle et al. 1999; Mikheeva et al. 2001).

The cluster mass (temperature) function and its evolution in $\Lambda$ CDM has been discussed in detail (e.g. Viana \& Liddle 1998; Bode et al. 2000; Henry 2000). To achieve a better consistency with cluster evolution data the value of $\Omega_{\mathrm{m}}$ should be lower than that obtained from the cluster number density test at $z=0$.

The dependence of the cluster mass (temperature) function on $z$ originates due to different growth rate of density perturbations in DM models. It is reflected in the appearance of the suppression factor $g\left(\Omega_{\mathrm{m}}(z)\right)$ in models with $\Omega_{\Lambda} \neq 0$ (see Eqs. (3), (4)):

$\sigma(R, z)=\frac{\sigma_{R}}{1+z} \frac{g\left(\Omega_{\mathrm{m}}(z)\right)}{g\left(\Omega_{\mathrm{m}}\right)}$

Below, we consider the cosmological models containing massive neutrino. In this case $z$-dependence is also present in the transfer function due to the free-streaming of hot matter particles (the effective growth rate becomes scale dependent). For the mass estimation of rich galaxy clusters (see Eq. (6)), the effect of free-streaming is negligible.

\section{Results and discussion}

\subsection{MDM models with non-zero tensor mode}

To test MDM models with a zero cosmological constant we follow the normalization procedure given by Arkhipova et al. (1999), Mikheeva et al. (2001). All models are normalized by $\sigma_{8}$ by the best fit present day cluster mass (Bahcall \& Cen 1993; Bahcall et al. 1997) and temperature (Henry \& Arnaud 1991; Donahue \& Voit 1999) functions. To achieve an agreement with $\Delta T / T$ data (Bennet et al. 1996) the contribution of cosmic gravitational waves is required. The value of the derived parameter $T / S$ depends mainly on the spectral index $n$, the abundance of hot matter $\Omega_{\nu}$, and the Hubble constant $h$.

Figure 1 shows the present-day cluster temperature functions $N(>T)$ for different values of $\Omega_{\nu}$ in MDM models with CGW normalized by $\sigma_{8} \simeq 0.52$. The normalization does not depend on $\Omega_{\nu}$, therefore all curves cross each other at some fixed point corresponding to the mass $M$ in the sphere of radius $8 h^{-1} \mathrm{Mpc}$.

A significant contribution of both CGW and massive neutrinos is needed to fit the large scale CMBA observational data. The consideration of the red spectra $(n<1)$ cannot reduce $T / S$ substantially. E.g., in MDM models with fixed $\Omega_{\nu}=0.2, h=0.6$, and $n=0.9,1,1.1$ : $T / S=0.6,1.7,3.6$ respectively, which strongly suppresses

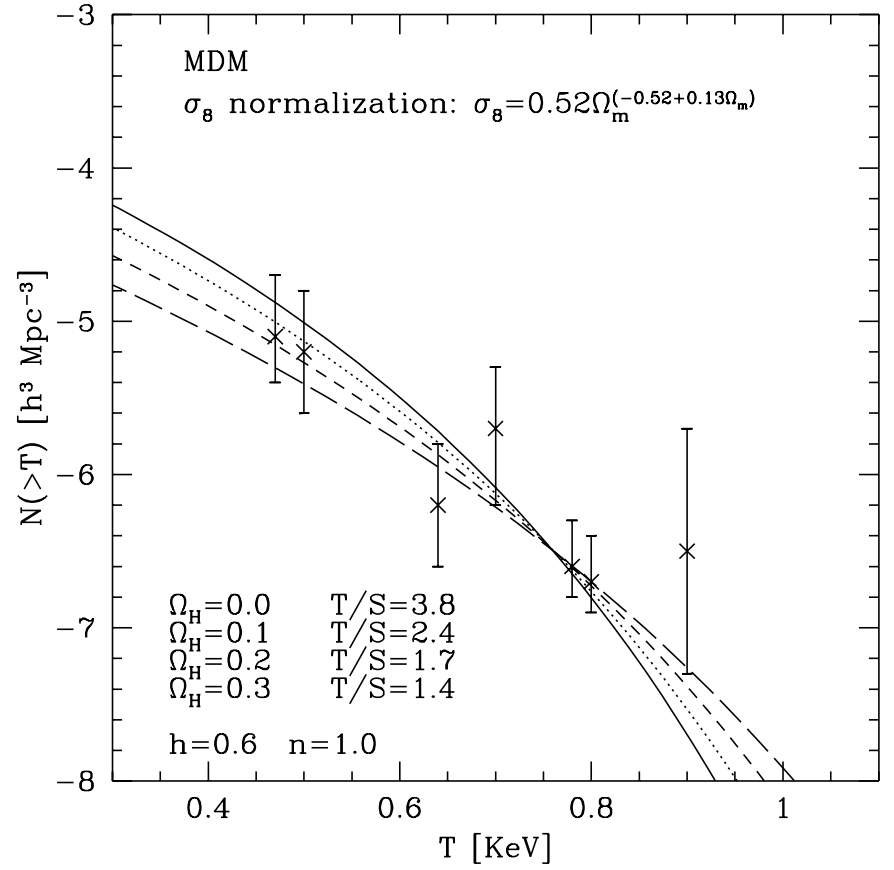

Fig. 1. The present day cluster abundance $N(>T)$ in MDM models normalized by $\sigma_{8}=0.52$ with $\Omega_{\nu}=$ $0,0.1,0.2,0.3$ (solid, dot, dash, long-dash lines, resp.), $\Omega_{\mathrm{b}}=$ $0.015 / h^{2}, h=0.6, n=1$. The needed $T / S$ is shown. The data points by Henry \& Arnaud (1991).

the height of the first acoustic peak in $\Delta T / T$ spectrum (Mikheeva et al. 2001).

In this respect, higher values of $\Omega_{\nu}$ would be preferable for MDM models: an increase of $\Omega_{\nu} \geq 0.2$ reduces the parameter $T / S$, however the problem arises with small-scale clustering and the $L y_{\alpha}$-cloud formation tests (Gnedin 1998). On the contrary, at low $\Omega_{\nu}<0.2$ neither possible changes of $h$ nor models with three species of massive neutrino can decrease sufficiently a high contribution of CGW into CMBA at large angular scales. The latter obviously leads to a low height of the first acoustic peak inconsistent with the BOOMERANG observations (Netterfield et al. 2001). Figure 2 demonstrates that the introduction of even a small cosmological constant would drastically improve the situation with MDM-CGW model parameters.

But let us return again to MDM models without $\Lambda$-term. Figure 3 presents the evolution of galaxy clusters. None of the considered models can fit the data at high $z>0.3$. We varied $h$ (Fig. 3a), $\Omega_{\nu}$ (Fig. 3b), and $n$ (not shown). The evolution tracks are practically independent of the post-inflationary spectral index for $n \in(0.9,1.1)$. Even neglecting the observational problems with $\mathrm{Ly}_{\alpha}$-forest, the case with high $\Omega_{\nu} \geq 0.2$ cannot help to achieve an agreement with the evolution of galaxy clusters in MDM dominated models. All the models predict the number of galaxy clusters at high $z \geq 0.3$ at least two orders of magnitude smaller than the observed one. This fact indicates that the evolution of density perturbations should be slower than that found in MDM without a cosmological constant. 

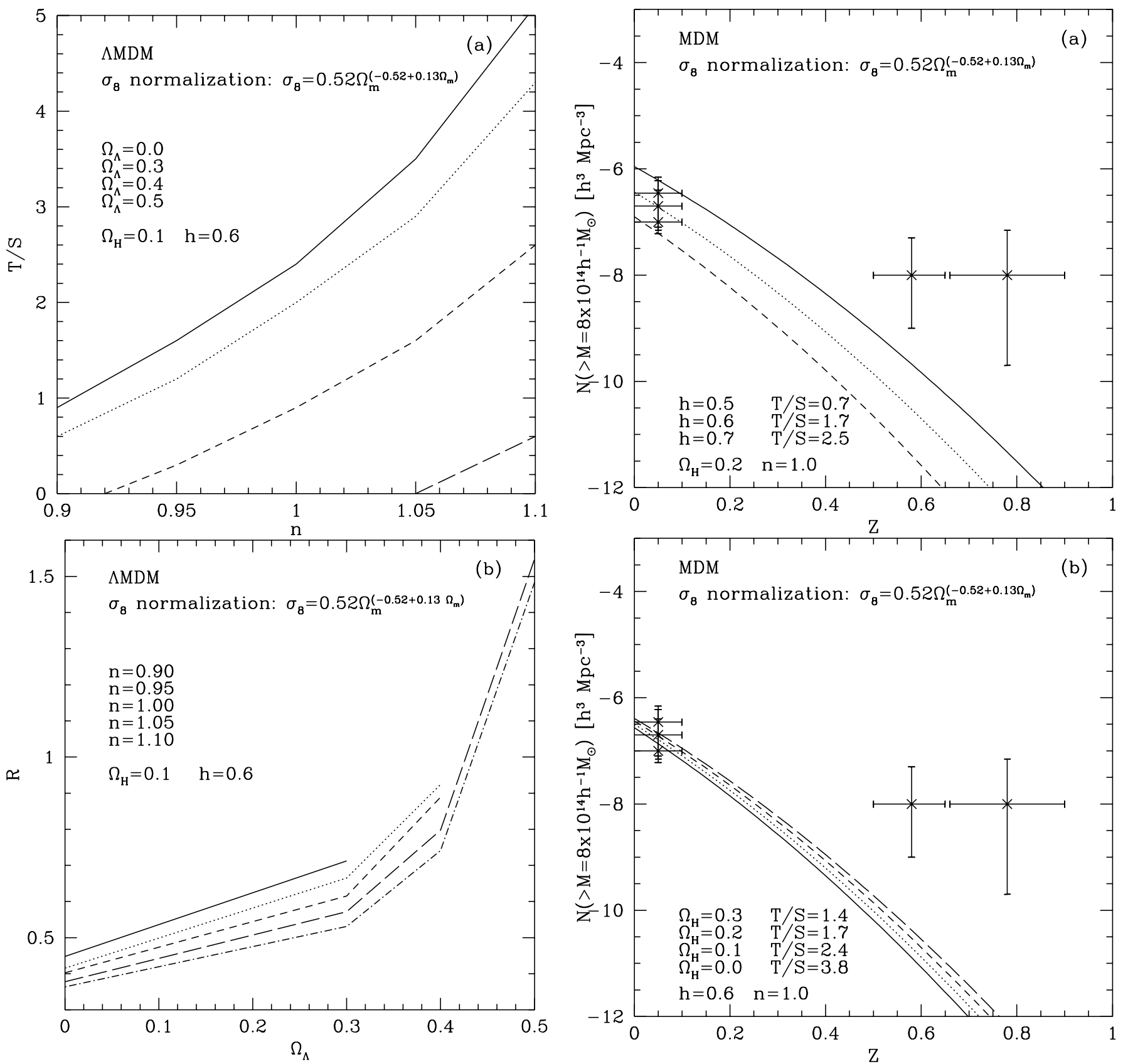

Fig. 2. The introduction of a cosmological constant in MDM-CGW models normalized by $\sigma_{8}=0.52 \Omega_{\mathrm{m}}^{\left(-0.52+0.13 \Omega_{\mathrm{m}}\right)}$ with $\Omega_{\nu}=0.1, \Omega_{\mathrm{b}}=0.015 / h^{2}, h=0.6$ : a) $T / S$ as a function of $n$ for $\Omega_{\Lambda}=0,0.3,0.4,0.5$ (solid, dot, dash, long-dash lines, resp.), and $\mathbf{b}$ ) the height of the first acoustic peak, $R^{\frac{1}{2}}=\frac{\delta T_{\mathrm{p}}}{70 \mu \mathrm{K}}$, as a function of $\Omega_{\Lambda}$ for $n=0.9,0.95,1,1.05,1.1$ (solid, dot, dashed, long-dashed, dashed-dot lines, resp.).

\subsection{MDM models with non-zero cosmological constant}

Let us consider flat $\Lambda \mathrm{MDM}$ models without CGW normalized by the COBE 4-year data. This allows an explicit derivation of $\sigma_{8}(\mathrm{cmb})$ as a function of model parameters. On the other hand, from observational abundance of galaxy clusters it is possible to obtain $\sigma_{8}(\mathrm{cl})$ as a function of $\Omega_{\mathrm{m}}$, e.g. $\sigma_{8}(\mathrm{cl})=0.52 \Omega_{\mathrm{m}}^{-0.52+0.13 \Omega_{\mathrm{m}}}$

Fig. 3. The cluster evolution $N\left(>M=8 \times 10^{14} M_{\odot}, z\right)$ in MDM models normalized by $\sigma_{8}=0.52$ with $n=1$, a) $\Omega_{\nu}=0.2, h=0.5,0.6,0.7$ (solid, dot, dash lines, resp.), and b) $h=0.6, \Omega_{\nu}=0,0.1,0.2,0.3$ (solid, dot, dash, longdash lines, resp.). The needed $T / S$ is shown. The data points by Bahcall \& Fan (1998).

(Eke et al. 1996; Liddle et al. 1996b). The coincidence between $\sigma_{8}(\mathrm{cmb})$ and $\sigma_{8}(\mathrm{cl})$ would limit the parameter $\Omega_{\Lambda}$ by $\sim 0.7$ in $\Lambda$ CDM (Bahcall \& Fan 1998; Henry 2000) and $\sim 0.5-0.6$ in $\Lambda$ MDM (Valdarnini et al. 1998; Novosyadlyj et al. 1999b) models.

According to Henry (2000) the cluster evolution test has a stronger upper-limited parameter $\Omega_{\mathrm{m}}$ in $\Lambda \mathrm{CDM}$ models than does the cluster abundance test at $z=0$. As an example we demonstrate the evolution 

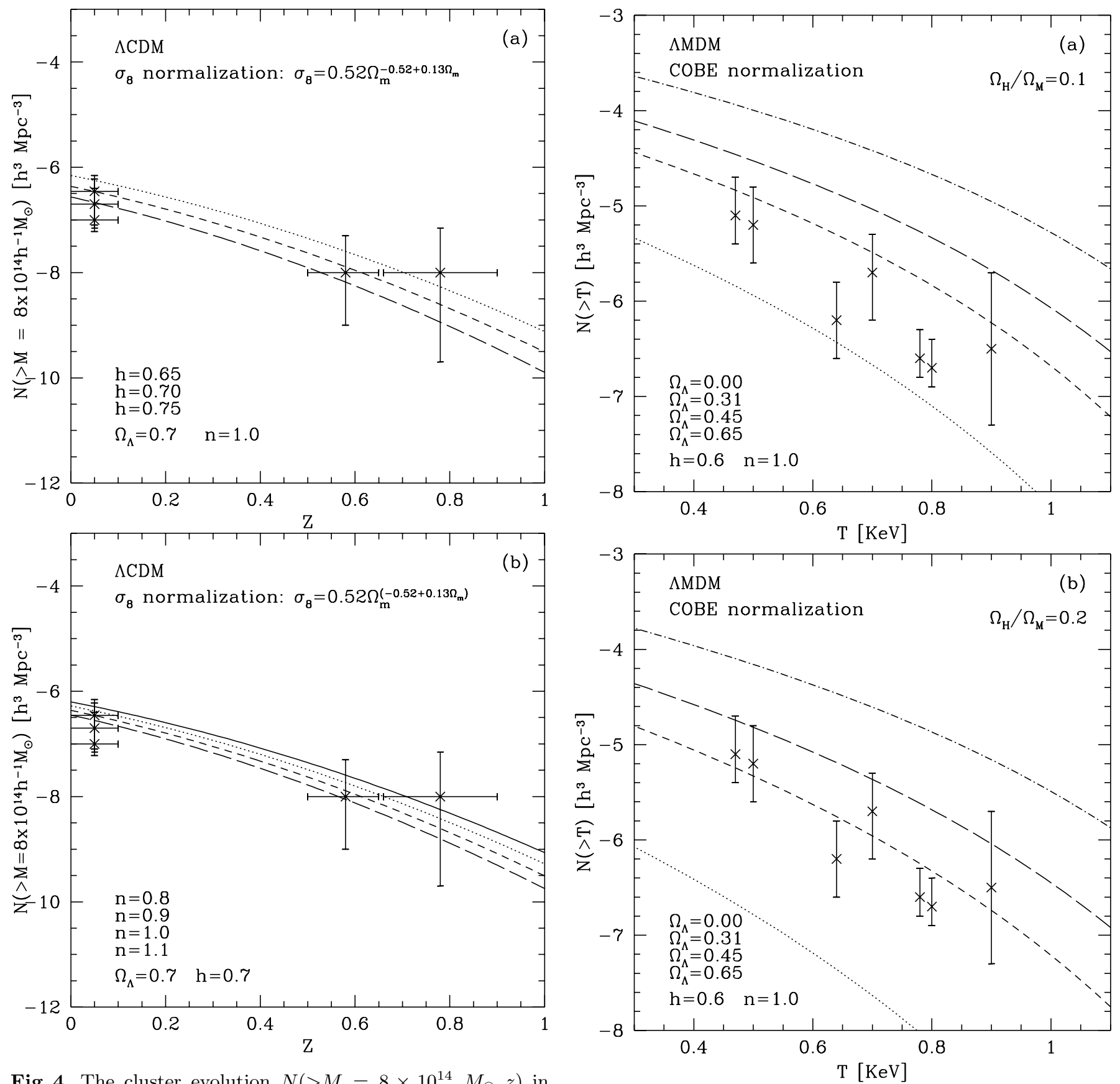

Fig. 4. The cluster evolution $N\left(>M=8 \times 10^{14} M_{\odot}, z\right)$ in $\Lambda$ CDM models normalized by $\sigma_{8}=0.52 \Omega_{\mathrm{m}}^{\left(-0.52+0.13 \Omega_{\mathrm{m}}\right)}$ with $\Omega_{\Lambda}=0.7, \Omega_{\mathrm{b}}=0.015 / h^{2}$, a) $n=1, h=0.65,0.70,0.75$ (dot, dash, long-dash lines, resp.), and b) $h=0.7, n=$ 0.8, 0.9, 1.0, 1.1 (solid, dot, dash, long-dash lines, resp.). The data points by Bahcall \& Fan (1998).

of the number density of galaxy clusters of mass $M \geq$ $8 \times 10^{14} M_{\odot}$ within the comoving radius $1.5 h^{-1} \mathrm{Mpc}$ in $\Lambda \mathrm{CDM}$ model with $\Omega_{\Lambda}=0.7$, as functions of $h$ (Fig. 4a) and $n$ (Fig. $4 \mathrm{~b}$ ). The cluster evolution is not practically affected by changes of the spectral index. Regarding the parameter $h$, the models with $h>0.6$ are preferable.

While the requested value of cosmological constant in $\Lambda$ CDM models is quite high, a small amount of hot

particles in $\Lambda \mathrm{MDM}$ models leads to the agreement with observations for a smaller $\Omega_{\Lambda}$.

We performed the calculation of functions $N(>T)$ for different parameters $\Omega_{\Lambda}$ and $f_{\nu}$ (Fig. 5). As we can see, to achieve an agreement with today cluster temperature function, we need a lower value of cosmological constant in the models with a higher fraction of hot matter. For 

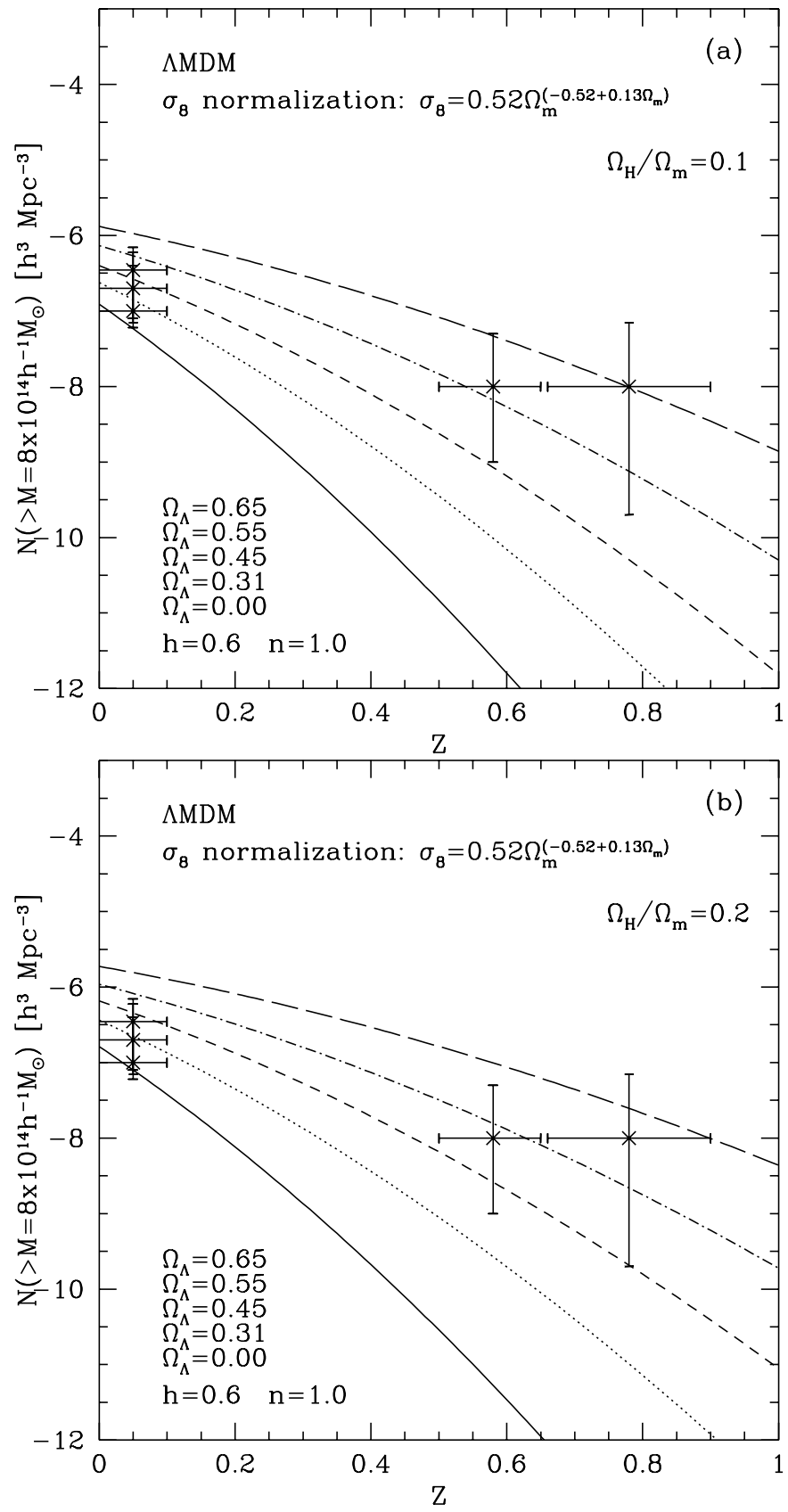

Fig. 6. The cluster evolution $N\left(>M=8 \times 10^{14} M_{\odot}, z\right)$ in $\Lambda$ MDM models normalized by $\sigma_{8}=0.52 \Omega_{\mathrm{m}}^{\left(-0.52+0.13 \Omega_{\mathrm{m}}\right)}$ with $\Omega_{\Lambda}=0,0.31,0.45,0.55,0.65$ (solid, dot, dash, dot-dash, longdash lines, resp.), $\Omega_{\mathrm{b}}=0.015 / h^{2}, h=0.6, n=1$, and $f_{\nu}=0.1$ a), 0.2 b). The data points by Bahcall \& Fan (1998).

$f_{\nu} \in(0,0.2), h \in(0.6,0.7), n \in(0.9,1.1)$, the value of cosmological constant remains in the range $(1 \sigma \mathrm{CL})$ :

$0.35<\Omega_{\Lambda}<0.7$.

Taking into account the $\mathrm{Ly}_{\alpha}$ forest test, models with $f_{\nu} \simeq 0.1$ are preferable. In these case parameter $\Omega_{\Lambda}$ is limited by $0.45 \leq \Omega_{\Lambda}<0.65$ (in agreement with some other results, cf. Valdarnini et al. 1998; Novosyadlyj et al. 1999b; Primack \& Gross 2000; Durrer \& Novosyadlyj 2001).

To obtain the redshift evolution functions of galaxy cluster abundance, the normalization of the power
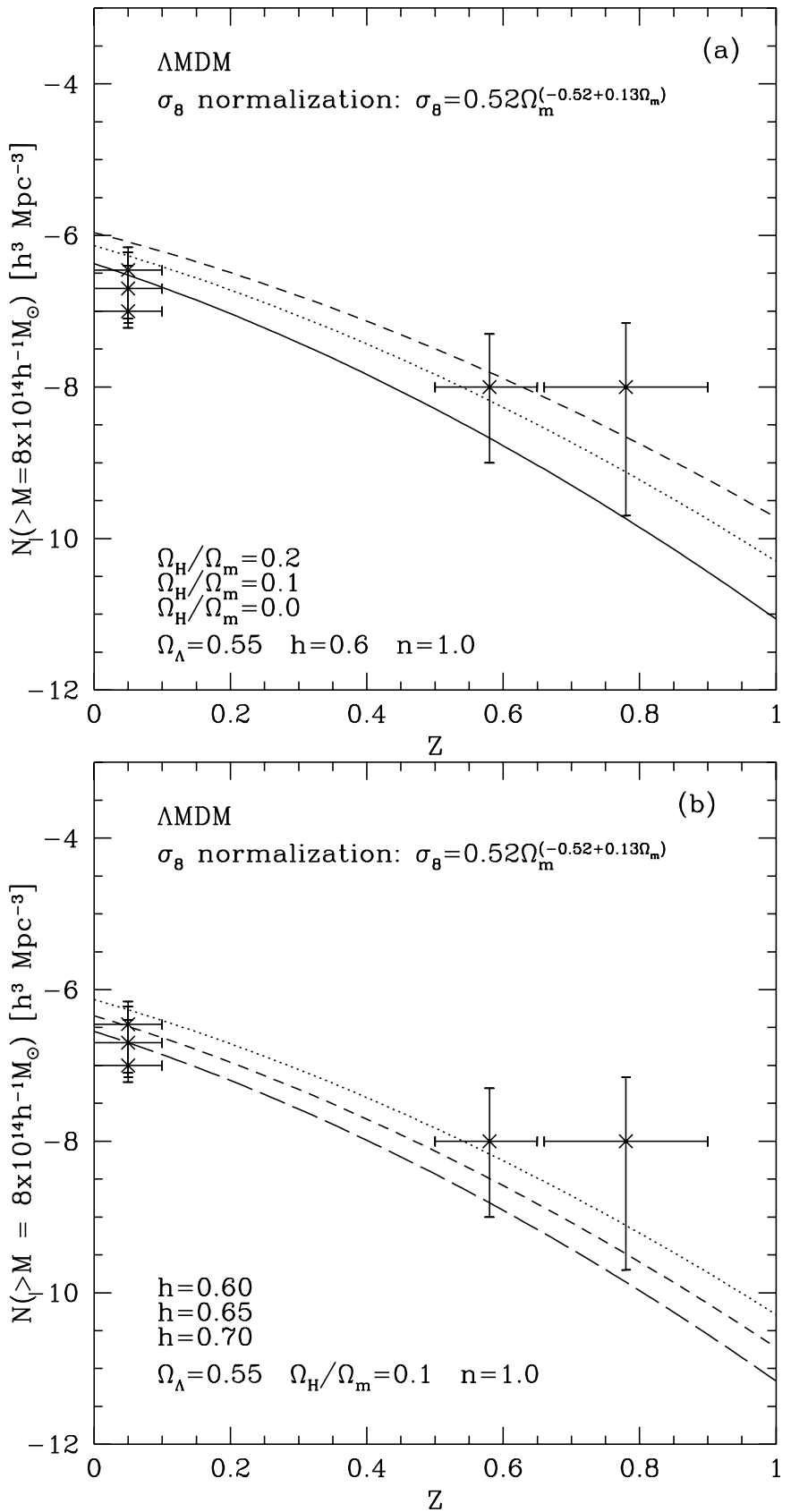

Fig. 7. The cluster evolution $N\left(>M=8 \times 10^{14} M_{\odot}, z\right)$ in $\Lambda \mathrm{MDM}$ models normalized by $\sigma_{8}=0.52 \Omega_{\mathrm{m}}^{-0.52+0.13 \Omega_{\mathrm{m}}}$ with $\Omega_{\Lambda}=0.55, \Omega_{\mathrm{b}}=0.015 / h^{2}, n=1$, a) $h=0.6, f_{\nu}=0,0.1,0.2$ (solid, dot, dash lines, resp), and b) $f_{\nu}=0.1, h=0.6,0.65,0.7$ (dot, dash, long-dash lines, resp.). The data points by Bahcall \& Fan (1998).

spectrum is done according to the present-day cluster concentration, $\sigma_{8}(\mathrm{cl})$, which depends on $\Omega_{\Lambda}$. At $1 \sigma$ level the best fit for $\sigma_{8}(\mathrm{cl})$ is not sensitive to the hot particle abundance, $h \in(0.6,0.7)$, and $n \in(0.9,1.1)$. Figures 6,7 present the galaxy cluster evolution in $\Lambda$ MDM models as function of parameters $\Omega_{\Lambda}, f_{\nu}$, and $h$. Changing $h$ and $n$ (within their ranges) does not practically influence the evolution tracks. Increasing $f_{\nu}$ reduces the needed value of $\Omega_{\Lambda}$ (in agreement with the cluster number density test). 
For $h \in(0.6,0.7), n \in(0.9,1.1)$, the value of $\Omega_{\Lambda}$ depends mainly on the fraction of hot matter $f_{\nu}$. We find the following approximation between the parameters $\Omega_{\Lambda}$ and $f_{\nu} \in(0,0.2)$ from the cluster redshift evolution alone $(1 \sigma \mathrm{CL})$ :

$\Omega_{\Lambda}+0.5 f_{\nu}=0.65 \pm 0.1$.

In connection with this degeneracy two points should be emphasized.

* As we see, the redshift evolution test slightly exaggerates the value of $\Omega_{\Lambda}$ in comparioson with the present day cluster abundance (cf. Eq. (10)). To be in the agreement with both tests the value of the cosmological constant in $\Lambda \mathrm{MDM}$ models should be found as $\Omega_{\Lambda} \in(0.45,0.7)$;

* Equation (11) can also be understood as a proportionality between the values of $\Omega_{\mathrm{m}}$ and $f_{\nu}$ : more matter leads to a greater fraction of hot matter and vice verce. Such a degeneracy is physically clear and could be straightforwardly observed in LSS tests. E.g., Novosyadlyj' et al. (1999b) indicate the correlation between $\Omega_{\mathrm{m}}$ and $\Omega_{\nu}$ from the cluster power spectrum and $\mathrm{Ly}_{\alpha}$ data. We guess that a joint LSS analysis will confirm Eq. (11) with a better precision.

In conclusion, we stress that the present observational data constrain only the parameter $f_{\nu} \in(0,0.2)$, the number of species of massive neutrino $\left(N_{\nu}=1,2,3\right)$ remains undetermined.

\section{Conclusions}

Our main conclusions are as follows:

- The introduction of massive neutrinos in spatially flat dark matter models affects drastically the estimation of the cosmological parameters from galaxy cluster observational data.

- MDM models with cosmic gravitational waves and a negligible $\Lambda$-term do not pass the observational constraints: they require a significal contribution of both (i) $\mathrm{CGW}(T / S \gtrsim 1)$ and (ii) hot matter $\left(f_{\nu} \gtrsim 0.2\right)$. The first condition reduces the first acoustic peak in $\Delta T / T$ spectrum by a level inconsistent with the BOOMERANG measurements, the second condition contradicts the $\mathrm{Ly}_{\alpha}$-system formation test.

- The introduction of even a small cosmological constant in MDM-CGW models essentially improves the situation with the model parameters, indicating that $\Lambda$-term is a more powerful instrument (than $T / S$ ) to reconcile theory with observations.

- The cluster abundance and/or evolution tests alone clearly hint at the existence of a non-zero cosmological constant in a set of spatially flat $\Lambda \mathrm{MDM}$ models with a negligible amount of CGW. A rise of $T / S$ cannot cancel the $\Lambda$-term. However, the opposite is not true: the estimation of $T / S$ in $\Lambda \mathrm{MDM}$ cosmologies requires better data and a more subtle analysis.

- The presence of a small fraction of massive neutrino, $f_{\nu} \in(0,0.2)$, (i) reduces the required value of cosmological constant (in comparison with $\Lambda \mathrm{CDM}$ ), (ii) is preferable from the point of view of the $\mathrm{Ly}_{\alpha}$ forest test, and (iii) does not discriminate the estimate of other cosmological parameters, $h \in(0.6,0.7), n \in(0.9,1,1)$, $N_{\nu}=1,2,3$.

- For $f_{\nu} \in(0,0.2), h \in(0.6,0.7), n \in(0.9,1.1)$, the value of cosmological constant remains in $1 \sigma$ ranges: $* \Omega_{\Lambda} \in(0.35,0.7)$ (from nearby cluster abundance), $* \Omega_{\Lambda} \in(0.45,0.75)$ (from cluster redshift evolution), $* \Omega_{\Lambda} \in(0.45,0.7)$ (from the both tests).

- We find the following approximation between parameters $\Omega_{\Lambda}$ and $f_{\nu} \in(0,0.2)$ from the cluster redshift evolution alone:

$$
\Omega_{\Lambda}+0.5 f_{\nu}=0.65 \pm 0.1, \quad(1 \sigma \mathrm{CL}) .
$$

- If massive neutrinos constitute only $\sim 10 \%$ of the total DM density $\left(f_{\nu}=0.1\right)$ the models with $\Omega_{\Lambda} \in$ $(0.5,0.65)$ satisfy the observational data best.

It may well be that the DM nature is more complex than just $\Lambda \mathrm{MDM}$ with a negligible amount of CGW. Moreover, even in the framework of this simple model we cannot delimit today the fraction and the number of species of massive neutrino, the accuracy of the available data is still low to do it. Nevertheless, we can assert that the abundance of cosmological massive neutrinos is small, $f_{\nu}<0.2$, corresponding less than few eV to the neutrino mass. Also, today we can conclude that

* The value of the estimated cosmological constant is very sensitive to even a small fraction of massive neutrinos, and

* the continuing progress in LSS cosmological observations assists in solving the DM problem.

Acknowledgements. The work of N.A.A. and V.N.L. was partly supported by RFBR (01-02-16274, 02-02-06368), INTAS (97-1192), and FST Program in Astronomy. The work of T.K. was supported by the COBASE program of USNRC.

\section{References}

Andres, E., Askebjer, P., Bai, X., Barouch, G., et al. 2000, Nucl. Phys. Proc. Suppl., 91, 423 [astro-ph/0009242]

Arhipova, N. A., Lukash, V. N., \& Mikheeva, E. V. 1999, Gravitational \& Cosmology, 5, 159 [astro-ph/9803328]

Bahcall, N. A., \& Cen, R. 1993, ApJ, 407, L49

Bahcall, N. A., Fan, X., \& Cen, R. 1997, ApJ, 485, 53

Bahcall, N. A., \& Fan, X. 1998, ApJ, 504, 1 
Bardeen, J. M., Bond, J. R., Kaiser, N., \& Szalay, A. S. 1986, ApJ, 304, 15

Bennett, C. L., Banday, A. J., Gorski, K. M., et al. 1996, ApJ, 464, L1

Bode, P., Bachall, N., Ford, E. B., \& Ostriker, J. P. 2000 [astro-ph/0011376]

Bond, et al. 2000 [astro-ph/0005004]

Bonometto, S. A., \& Pierpaoli, E. 1998, NewA, 3, 391 [astro-ph/9806035]

Bridle, S. L., Eke, V. R., Lahav, O., et al. 1999, MNRAS, 310, 565

Bunn, E. F., \& White, M. 1997, ApJ, 480, 6

Carroll, S. M., Press, W. H., \& Turner, E. L. 1992, A\&A, 30, 499

Donahue, M., \& Voit, G. M. 1999, ApJ, 523, L137

Durrer, R., \& Novosyadlyj, B. S. 2001, MNRAS, 324, 560 [astro-ph/0009057]

Efstathiou, G., Frenk, C. S., White, S. D. M., \& Davis, M. 1988, MNRAS, 235, 715

Eisenstein, D., \& Hu, W. 1998, ApJ, 496, 605

Eke, V. R., Cole, S., \& Frenk, C. S. 1996, MNRAS, 282, 263

Eke, V. R., Navarro, J., \& Frenk, C. S. 1998, ApJ, 503, 569

Fang, L. Z., Xiang, S. P., \& Li, S. X. 1984, A\&A, 140, 77

Feast, M. W., \& Catchpole, R. W. 1997, MNRAS, 286, L1

Gardini, A., Bonometto, S. A., \& Murante, G. 1999, ApJ, 524, 510

Gawiser, E. 2000 [astro-ph/0005365]

Gnedin, N. Y. 1998, MNRAS, 299, 393

Gross, M. A., Somerville, J. R., Primack, J. R., Borgani, S., \& Girardi, M. 1998, Large-Scale Structure: Tracks and Traces, Proc. 12th Potsdam Cosmology Workshop, Potsdam, Sep. 15-19, ed. V. Mueller, J. P. Gottloeber, \& J. Muecket (World Scientific), 171

Henry, J. P. 2000, ApJ, 534, 565

Henry, J. P., \& Arnaud, K. A. 1991, ApJ, 372, 410

Holtzman, J. A. 1989, ApJS, 71, 1

Hu, W., Fukugita, M., Zaldarriaga, M., \& Tegmark, M. 2000 [astro-ph/0006436]

Jaffe, A. H., Ade, P. A., Balbi, A., et al. 2001, Phys. Rev. Lett., 86,3475

Kahniashvili, T. A., Novosyadlyj, B. S., \& Valdarnini, R. 1996, Phys. Hel. Acta, 71, 454

Klypin, A., Primack, J., \& Holtzman, J. 1996, ApJ, 466, 13

Kochanek, C. S. 1996, ApJ, 466, 638

Kofman, L. A., Gnedin, N. Y., \& Bahcall, N. A. 1993, ApJ, 413, 1

Kofman, L. A., \& Starobinsky, A. A. 1985, Sov. Astron. Lett., 9,643

Komberg, B. V., Kravtsov, A. V., \& Lukash, V. N. 1996, MNRAS, 282, 713

Lacey, C., \& Cole, S. 1994, MNRAS, 271, 676

Liddle, A. R., Lyth, D. M., Roberts, D., \& Viana, P. 1996a, MNRAS, 278, 644
Liddle, A. R., Lyth, D. M., Viana, P., \& White, M. 1996b, MNRAS, 282, 281

Locas, E. L., \& Hoffman, Y. 2000 [astro-ph/0011295]

Lucchin, F., Colafrancesco, S., de Gasperis, G., et al. 1996, ApJ, 459, 455

Lukash, V. N. 1991, Annals New York Acad. of Sci., 647, 659

Lukash, V. N., \& Mikheeva, E. V. 2000, Int. J. Mod. Phys. A, 15,3783

Ma, C.-P. 1996, ApJ, 471, 13

Melchiorri, A., Sazhin, M. V., Shulga, V. V., \& Vittorio, N. 1999, ApJ, 518, 562

Mikheeva, E. V., Lukash, V. N., Arkhipova, N. A., \& Malinovsky, A. M. 2001, Astron. Zh., 78, 195 (Astron. Rep., $45,163)$

Navarro, J. F., Frenk, C. S., \& White, S. D. M. 1995, MNRAS, 275,720

Netterfield, C. B., et al. 2001 [astro-ph/0104460]

Novikov, I. D., \& Zel'dovich, Ya. B. 1975, Structure and Evolution of the Universe (Nauka, in Russian)

Novosyadlyj, B. S., Durrer, R., \& Lukash, V. N. 1999a, A\&A, 347, 799

Novosyadlyj, B. S., Durrer, R., Gottlober, S., Lukash, V. N., \& Apunievych, S. 1999b, A\&A, 356, 418

Padmanabhan, T. 1993, Structure Formation in the Universe (Cambridge Univ. Press)

Peacock, J. A. 1997, MNRAS, 284, 885

Peebles, P. J. E. 1984, ApJ, 284, 439

Perlmutter, S., Aldering, G., Goldhaber, G., et al. 1999, ApJ, 517,565

Pogosyan, D. Yu., \& Starobinsky, A. A. 1995, ApJ, 447, 465

Press, W. H., \& Schechter, P. 1974, ApJ, 187, 425

Primack, J. R., \& Klypin, A. 1996, Nucl. Phys. Proc. Suppl., $51 \mathrm{~B}, 30$

Primack, J. R., \& Gross, M. A. 2000 [astro-ph/0007165]

Rahman, N., \& Shandarin, S. 2001 [astro-ph/0010228]

Rubakov, V. A., Sazhin, M. V., \& Verjaskin, A. M. 1982, Phys. Lett. B, 115, 189

Seljak, U., \& Zaldarriaga, M. 1996, ApJ, 469, 437

Shafi, Q., \& Stecker, F. W. 1984, Phys. Rev. Lett., 53, 1292

Smoot, G. F., Bennett, C. L., Kogut, A., et al. 1992, ApJ, 396, L1

Starobinsky, A. A. 1979, JETF Lett., 30, 719

Tegmark, M. 1999, ApJ, 514, L69

Valdarnini, R., \& Bonometto, S. A. 1984, Phys. Lett. A, 103, 369

Valdarnini, R., Kahniashvili, T. A., \& Novosyadlyj, B. S. 1998, A\&A, 311, 1

Viana, P. T. P., \& Liddle, A. R. 1996, MNRAS, 281, 323

Viana, P. T. P., \& Liddle, A. R. 1998, MNRAS, 303, 535

Wu, J.-H.P. 2000 [astro-ph/0012207]

Zakharov, A. V. 1979, JETP, 77, 434 (in Russian) 